\title{
Erratum zu: Regionalisierung hydrologischer Modelle mit Function Space Optimization
}

\author{
Moritz Feigl (D) - Mathew Herrnegger (D) - Robert Schweppe $(\mathbb{D}) \cdot$ Stephan Thober · Daniel Klotz (D) \\ Luis Samaniego (iD) $\cdot$ Karsten Schulz (iD
}

Online publiziert: 17. Juni 2021

(C) Österreichischer Wasser- und Abfallwirtschaftsverband (ÖWAV) and Springer-Verlag GmbH Austria, ein Teil von Springer Nature 2021

\section{Erratum zu:}

Österr Wasser- und Abfallw 2021

https://doi.org/10.1007/s00506-02100766-0

Im Originalbeitrag wurde ein Co-Autor vergessen: Dr. Luis Samaniego, Department Hydrosystemmodellierung, UFZ-Helmholtz Zentrum für Umweltforschung, Permoserstraße 15, 04318, Leipzig, Deutschland.

Der Originalbeitrag wurde korrigiert.

Die Online-Version des

Originalartikels ist unter https://doi. org/10.1007/s00506-021-00766-0 zu finden.

DI M. Feigl $(\bowtie) \cdot$ DI Dr. M. Herrnegger • Univ.-Prof. Dipl.-Geoökol. Dr. K. Schulz Institut für Hydrologie und Wasserwirtschaft, Universität für Bodenkultur Wien,

Muthgasse 18, 1190 Wien, Österreich moritz.feigl@boku.ac.at

R. Schweppe, M.Sc. • Dr. S. Thober • Dr. L. Samaniego

Department

Hydrosystemmodellierung,

UFZ-Helmholtz Zentrum

für Umweltforschung,

Permoserstraße 15, 04318 Leipzig,

Deutschland

DI Dr. D. Klotz

LIT AI Lab \& Institute für Machine Learning, Johannes Kepler Universität

Linz, Altenberger Straße 69, 4040 Linz, Österreich 